\title{
The Optimization Variables of Input Data of Artificial Neural Networks for Diagnosing Acute Appendicitis
}

\author{
Sung Yun Park and Sung Min Kim* \\ Department of Medical Biotechnology, Dongguk University - Seoul, 100715 Seoul, Republic of Korea
}

Received: 25 Jun. 2013, Revised: 4 Nov. 2013, Accepted: 6 Nov. 2013

Published online: 1 Jan. 2014

\begin{abstract}
The purpose of this study is to suggest an efficient diagnosis system for acute appendicitis using the artificial neural network model with optimized input variables. Acute appendicitis is one of the most common diseases of the abdomen. However, the accuracy of diagnosis is not high even with experienced surgeons due to its complex symptoms. We used the artificial neural networks model to analyze the complex problems. A total of 801 suspected acute appendicitis patients were collected and a multilayer neural network with thirteen input variables, and two hidden layers with thirty neurons were used to diagnosis acute appendicitis. The mean-square error (0.0011) was stabilized after seven input variables. The nine to thirteen input variables had a high and equal performance $(98.81 \%$, $100 \%, 98.39 \%, 100 \%, 99.31 \%$, and 0.995 for specificity, sensitivity, positive predictive value, negative predictive value, accuracy and AUC, respectively). We had optimized the input variables and the performance is significantly higher than the published diagnosis method such as the Alvarado clinical scoring system. We believe that the developed model regarding the multilayer neural network would be a useful method to rapidly and correctly diagnosis acute appendicitis for clinical surgeons.
\end{abstract}

Keywords: Acute appendicitis, artificial neural network, mean-square errors, area under an ROC curve

\section{Introduction}

Acute appendicitis is one of the most common diseases of the abdomen in emergency departments. The incidence of acute appendicitis is almost $7 \%$ for males and females $[1,2]$. The treatment for acute appendicitis usually commonly requires a surgical method. The negative or delays of diagnosis for acute appendicitis lead to an increase in the morbidity and mortality rates [3]. Unfortunately, a clinical diagnosis for acute appendicitis is not easy even for experienced surgeons.

Several methods for diagnosing acute appendicitis have been developed to increase the accuracy rate of diagnosis and to decease the negative appendectomy rate [4-6]. The initial method of diagnosing acute appendicitis have consisted of demographics, history, physical examination, and laboratory findings. In 1986, Alvarado proposed a clinical scoring system including 8 items with 10 maximum points [4]. Recently, image analysis techniques such as computed tomography (CT), ultrasound (US), and magnetic resonance imaging (MRI) have shown good performance as $93 \%$ to $98 \%[5,6]$. However, the clinical scoring system has shown poor diagnostic performance even in western societies.
Some researchers have developed and modified the Alvarado clinical scoring system, and they suggested the new clinical scoring systems such as the Lintula score, and RIPASA score $[7,8]$. The CT, and MRI are highly associated with radiation exposure and diagnostic quality of US highly depends on operator. First of all, image analysis techniques cannot use at out of work-time, especially in emergency department.

Artificial neural networks (ANNs) have been interesting as a computer-aided diagnostic system. Several researchers have shown good performance in various clinical fields including cancer prediction, and head injuries $[9,10]$. The basic structure of ANNs is imitating the central nervous system. The ANNs basically have three layers which are input, hidden, and output layer. Each layer estimates the input data from the previous layer, or output data to the next layer. There are weight factors between layers which are complicatedly connected with each node. As these elements, the ANNs have been usefully used a solving method in nonlinear problems.

The purpose of this study is to find the minimum number of input data of artificial neural networks for

\footnotetext{
*Corresponding author e-mail: smkim@dongguk.edu
} 


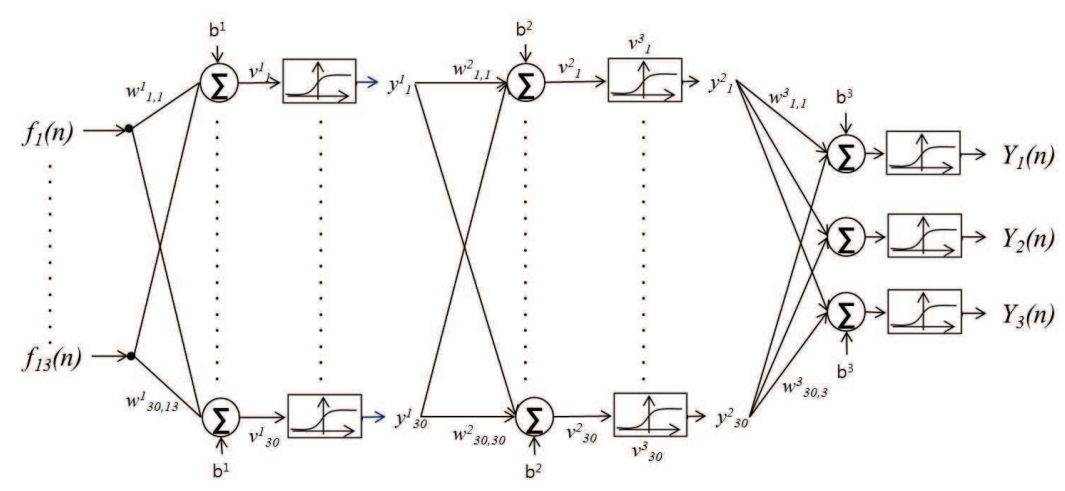

Fig. 1: A structure of multilayer neural networks with one input layer, two hidden layer, and one output layer.

diagnosing acute appendicitis. Then, we suggest a high efficient model of ANNs to diagnose acute appendicitis for emergency clinic surgeons.

\section{Patients data}

Patient who underwent abdominal pain have were gathered at an emergency department of Dongguk University hospital in South Korea from August 2011 to April 2012. The categories of the diagnosis case report were developed based on the World Organization of Gastroenterology Research Committee. The clinic doctors were regularly trained to keep uniform quality of the data set. Patients who felt abdominal pain were interviewed according to the procedures of the diagnosis case report then; suspected acute appendicitis patients took the CT, US or MRI. The patients were assorted into three groups; group 1 for No appendicitis (NA) patients, group 2 for Normal appendicitis (NorA) patients, and group 3 for Acute appendicitis (AA) patients. The group position of patients was decided by the final decision of surgeons. All protocols of this study were approved by the Institutional Review Board of Dongguk University Hospital.

\section{Artificial neural networks}

The patients data were randomly assigned into two classifications. Of patients data, $80 \%$ was used to

Table 1: Patients' characteristics

\begin{tabular}{cccc}
\hline & No appendicitis & \multicolumn{2}{c}{ Appendicitis $(n=380)$} \\
\cline { 3 - 4 }$(n=421)$ & $\begin{array}{c}\text { Normal } \\
\text { appendicitis } \\
(n=158)\end{array}$ & $\begin{array}{c}\text { Acute } \\
\text { appendicitis } \\
(n=142)\end{array}$ \\
\hline $\begin{array}{c}\text { Age (years) } \\
\text { Gender }\end{array}$ & $30.46 \pm 13.03$ & $31.95 \pm 12.09$ & $32.66 \pm 12.83$ \\
(male/female) & $135 / 286$ & $83 / 75$ & $92 / 50$ \\
\hline
\end{tabular}

construct the ANNs, and $20 \%$ was used to indicate the performance of ANNs. The multilayer neural network (MLNN) structure with two hidden layers was used for diagnosing acute appendicitis. The back-propagation (BP) leaning algorithm of MLNN was used in this study. The MLNN consists of one input layer with 413 features, two hidden layers with 30 neurons, and one output layer with an index of three classes as see figure 1.We have construct a structure of MLNN on reducing input datas variables stage by stage. Each layer consists of a summation part; and an activation part except the input layer. In this study, the summation function is a linear type as in equation (1), and the activation function is a nonlinear sigmoid type as in equation (2).

$$
\begin{aligned}
& \mathbf{v}_{i}^{1}=\left(\sum_{j=1}^{30} \mathbf{w}_{j, i}^{1} \times \mathbf{f}_{1}\right)+\left(b^{1}\right) \\
& \mathbf{y}_{j}^{1}=\left(1+\exp \left(-\mathbf{v}_{i}^{1}\right)\right)^{-1}
\end{aligned}
$$

where, $\mathbf{v}_{i}^{1}$ is the output of summation of the $i^{t h}$ neuron, $\mathbf{w}_{j, i}^{1}$ is the weight factor of the first hidden layer, $b^{1}$ is a bias of the first hidden layer, and $\mathbf{y}_{j}^{1}$ is the output of activation function of $j^{\text {th }}$ neuron in the first hidden layer. The structure of MLNN with the back-propagation learning algorithm was constructed using MATLAB (Mathworks, MA). In the order to investigate the performance of the new diagnosis method, we compared the Alvarado clinic scoring system and the developed method using ANNs. The Alvarado clinic scoring system has 9 items (migratory RIF pain for 1 point, anorexia for 1 point, nausea/vomiting for 1 , tenderness: right iliac fossa for 2, rebound tenderness RIF for 1 point, elevated temperature for 1 , leucocytosis for 2 point, and shift to the left of neutrophils for 1) with a total score of 10. If a score of 8 or above is acquired in the Alvarado clinic scoring system, the patients is under appendicitis [4]. 
Table 2: Comparison of performance between Alvarado clinical scoring systems and multilayer neural network(MLNN)

\begin{tabular}{|c|c|c|c|c|c|c|c|}
\hline \multicolumn{2}{|c|}{ Methods of diagnostic } & \multirow{2}{*}{$\begin{array}{c}\text { Specificity } \\
(\%)\end{array}$} & \multirow{2}{*}{$\begin{array}{c}\text { Sensitivity } \\
(\%)\end{array}$} & \multirow{2}{*}{$\begin{array}{c}\text { Positive } \\
\text { predictive } \\
\text { value }(\%)\end{array}$} & \multirow{2}{*}{$\begin{array}{l}\text { Negarive } \\
\text { predictive } \\
\text { value }(\%)\end{array}$} & \multirow{2}{*}{$\begin{array}{c}\text { Accuracy } \\
(\%)\end{array}$} & \multirow[b]{2}{*}{ AUC } \\
\hline type & $\begin{array}{c}\# \text { of } \\
\text { input variables }\end{array}$ & & & & & & \\
\hline \multirow{4}{*}{$\begin{array}{l}\text { Alvarado } \\
\text { (Clinical } \\
\text { scoring } \\
\text { system) }\end{array}$} & 9 & 88.00 & 28.26 & 57.14 & 68.42 & 66.40 & 0.672 \\
\hline & 13 & 98.81 & 100 & 98.39 & 100 & 99.31 & 0.995 \\
\hline & 12 & 98.81 & 100 & 98.39 & 100 & 99.31 & 0.995 \\
\hline & 11 & 98.81 & 100 & 98.39 & 100 & 99.31 & 0.995 \\
\hline \multirow{7}{*}{$\begin{array}{c}\text { MLNN } \\
\text { (Artificial } \\
\text { neural } \\
\text { networks) }\end{array}$} & 10 & 98.81 & 100 & 98.39 & 100 & 99.31 & 0.995 \\
\hline & 9 & 98.81 & 100 & 98.39 & 100 & 99.31 & 0.995 \\
\hline & 8 & 97.62 & 98.36 & 96.77 & 98.80 & 97.93 & 0.837 \\
\hline & 7 & 78.57 & 34.43 & 53.85 & 62.26 & 60.00 & 0.843 \\
\hline & 6 & 58.33 & 16.39 & 22.22 & 49.00 & 40.69 & 0.514 \\
\hline & 5 & 26.19 & 11.48 & 10.14 & 28.95 & 20.00 & 0.492 \\
\hline & 4 & 2.38 & 3.23 & 2.74 & 2.38 & 3.23 & 0.050 \\
\hline
\end{tabular}

\section{Statistical Analyses}

A receiver operating characteristics (ROC) graph, an area under an ROC curve (AUC), and the mean square error (MSE) was used to measure the performance of ANNs to diagnosis acute appendicitis [11]. The ROC graph is plotted by the true positive rate for the $\mathrm{x}$-axis, and the false positive rate for the y-axis. The shape of the upper right-hand curve means that the method has a good performance. The AUC has three zones which equal 1 for perfect performance, greater than 0.8 for good, between 0.6 and 0.8 for moderate, and less than 0.6 for poor.

\section{Results}

A total of 801 patients with an age of $31.47 \pm 13.35$ years (mean \pm SD (standard deviation)) were enrolled from August 2011 to April 2012 in seen table 1. Eighty per cent (337 patients for NA group, 126 patients for NorA group, and 113 patients for AA group) and twenty per cent (84 patients for NA group, 32 patients for NorA group, and 29 patients for AA group) of all patients were used to construct and to validate a structure of ANNs, respectively.

The variables of input data were decided based on the Alvarado clinical scoring system and previous literatures. The maximum mean-square error (2.843) was at four input variables (anorexia, nausea/vomiting, tenderness: right iliac fossa (RIF), and rebound tenderness RIF) and the minimum mean-square error (3.412E-4) was at thirteen input variables (age, sex, migratory RIF pain, anorexia, nausea/vomiting, tenderness: right iliac fossa, rebound tenderness RIF, bowel sound, abnormal wall rigidity, elevated temperature, leucocytosis, shift to the left of neutrophil, and CRP) as seen figure 2 .

The performance of the Alvarado clinical scoring system were $88 \%, 28.6 \%, 57.14 \%, 68.42 \%, 66.40 \%$, and
0.672 for specificity, sensitivity, positive predictive value, negative predictive value, accuracy and AUC, respectively. The performances of ANNs were shown the same values at thirteen, twelve, eleven, ten, and nine input variables $(98.81 \%, 100 \%, 98.39 \%, 100 \%, 99.31 \%$, and 0.995 for specificity, sensitivity, positive predictive value, negative predictive value, accuracy and AUC, respectively) and the minimum performance of ANNs was measured at four input variables $(2.38 \%, 3.23 \%$, $2.74 \%, 2.38 \%, 3.23 \%$, and 0.05 for specificity, sensitivity, positive predictive value, negative predictive value, accuracy and AUC, respectively)

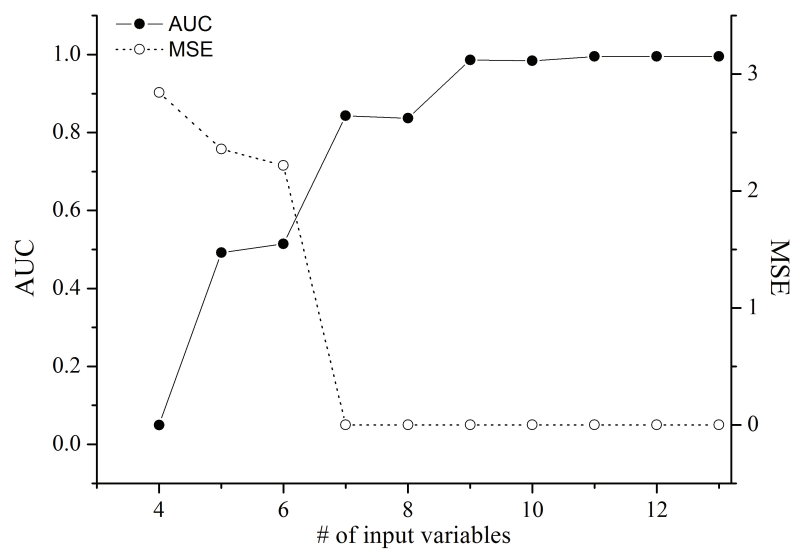

Fig. 2: The ratio of mean square error (MSE) and value of an area under an ROC curve (AUC) at numbers of input variables of MLNN 


\section{Discussion}

The Acute appendicitis is one of most common diseases in the emergency department of hospital [1-3]. Several methods were established such as the clinical scoring system, imaging analysis, and computer-aid [4-8]. However, the clinical diagnosis of appendicitis is not easy even for experienced surgeons due to its complex diagnosis process. The artificial neural networks are one of the useful methods to analyse a complex interrelation such as acute appendicitis. The purpose of this study, therefore, is to suggest suggesting highly efficient model of ANNs with small input variables to diagnose acute appendicitis for emergency clinic surgeons.

The mean-square error ratio was rapidly decreased from six to seven variables of input data. It means that the MLNN structure was stabilized after seven variables of input data. Indeed, the removal of five variables (age, sex, abnormal wall, bowel sound, and temperature) might not be essentially needed to diagnosis acute appendicitis using the MLNN method.

Previous studies reported the various performances to diagnose acute appendicitis using artificial neural networks [12-14]. Prabhudesai et al. reported high performances (100\% for sensitivity, $97.2 \%$ for specificity, $96.0 \%$ for positive predictive value, $100 \%$ negative predictive value) with eleven input variables [12]. Hsieh et al. [13], however, demonstrated poor performances (94\% for sensitivity, $85 \%$ for specificity, $94 \%$ for positive predictive value, $85 \%$ negative predictive value, and 0.91 for AUC) with ten input variables and Sakai et al. [14] also did not report good performance $(0.741 \pm 0.051$ for AUC) with nine input variables. Our results demonstrate a greater number of good performances even on eight input variables. We believe that the reasons might due to number of patients. We used 801 patients, whereas the study of Prabhudesai et al. [12], which has the best performance in previous studies, used 60 patients. Because the ANNs is pattern analysis method it demonstrated performance with larger input data set.

At four input variables, the performance is rapidly decreased as compared with the conditions of previous input variables. The condition of four input variables was removed a migratory RIF pain on previous condition. The reason might be that the migratory RIF pain is one of the main symptoms on acute appendicitis. However, when we intentional lose a migratory RIF pain variable, the performances do not change on the constructed model of MLNN with thirteen input variables. We think that the reason is a property of artificial neural networks which is fault tolerance.

Although we used data from over eight hundred patients', the patients were collected from only one local emergency department. To use in a different area, or country, the model of MLNN must be further developed and stabilized after using patients' data from different area, or county.

\section{Conclusion}

We developed an efficient model of MLNN with optimized input variables to diagnose acute appendicitis and this model demonstrated very good performance as compared with the clinical scoring system and previous studies. Further study is needed to evaluate the developed model of MLNN in more detail using pretty quantity patients. Finally, we believe that the developed model of MLNN would help clinical surgeons rapidly and accurately diagnose suspected acute appendicitis.

\section{Acknowledgement}

This study was supported by a grant of the Korea Healthcare Technology R\&D Project, Ministry of Health \& Welfare, Republic of Korea(A102058).

\section{References}

[1] A. Petroianu, Diagnosis of acute appendicitis, Int. J. Surg., 10, 115-19 (2012).

[2] H. John, U. Neff, M. Kelemen, Appendicitis diagnosis today: clinical and ultrasonic deductions, World J. Surg., 17, 243-49 (1993).

[3] J. Garfield, R. Birkhahn, T. Gaeta, W. Briggs, Diagnostic pathways and delays on route to operative intervention in acute appendicitis, Am. Surg., 70, 1010-13 (2004).

[4] A. Alvarado, A practical score for the early diagnosis of acute appendicitis, Ann Emerg Med., 15, 557-64 (1986).

[5] E. Paulson, C. Coursey, CT protocols for acute appendicitis: time for change, AJR Am. J. Roentgenol., 193, 1268-71 (2009).

[6] G. M. Israel, N. Malguria, S. McCarthy, J. Copel, J. Weinreb, MRI vs. ultrasound for suspected appendicitis during pregnancy, J. Magn. Reson. Imaging., 28, 428-33 (2008).

[7] H. Lintula, H. Kokki, J. Pulkkinen, R. Kettunen, O. Gröhn, M. Eskelinen, Diagnostic score in acute appendicitis. Validation of a diagnostic score (Lintula score) for adults with suspected appendicitis, Langenbecks Arch. Surg., 395, 495-500 (2010).

[8] C. Chong, M. Adi, A. Thien, A. Suyoi, A. Mackie, A. Tin, S. Tripathi, N. Jaman, K. Tan, K. Kok, V. Mathew, O. Paw, H. Chua, S. Yapp, Development of the RIPASA score: a new appendicitis scoring system for the diagnosis of acute appendicitis, Singapore Med. J., 51, 220-25 (2010).

[9] H. Abbass, An evolutionary artificial neural networks approach for breast cancer diagnosis, Artif. Intell. Med., 25, 265-81 (2002).

[10] O. Erab, N. Yumusakc, F. Temurtasa, Chest diseases diagnosis using artificial neural networks, Expert Syst. Appl., 37, 7648-55 (2010).

[11] Tom Fawcett, An introduction to ROC analysis, Pattern Recognit. Lett., 27, 861-74 (2006).

[12] S. Prabhudesai, S. Gould, S. Rekhraj, P. Tekkis, G. Glazer, P. Ziprin, Artificial neural networks: useful aid in diagnosing acute appendicitis, World J. Surg., 32, 305-09 (2008). 
[13] C. H. Hsieh, R. H. Lu, N. H. Lee, W. T. Chiu, M. H. Hsu, Y. C. Li, Novel solutions for an old disease: diagnosis of acute appendicitis with random forest, support vector machines, and artificial neural networks, Surgery., 149, 87-93 (2011).

[14] S. Sakai, K. Kobayashi, J. Nakamura, S. Toyabe, K. Akazawa, Accuracy in the diagnostic prediction of acute appendicitis based on the Bayesian network model, Methods Inf. Med., 46, 723-26 (2007).

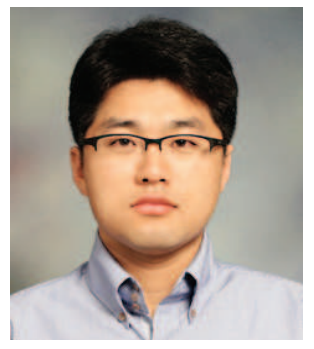

Sung Yun Park received the MS degree in biomedical engineering from Konkuk University in 2007, and the $\mathrm{PhD}$ degree in Med-bioengineering from the department of Bio technology engineering, Dongguk University in 2012. He is currently a research professor in Dongguk University. His research interests are in the areas of artificial neural networks, pattern recognition, biosignal analysis and animal experiment.

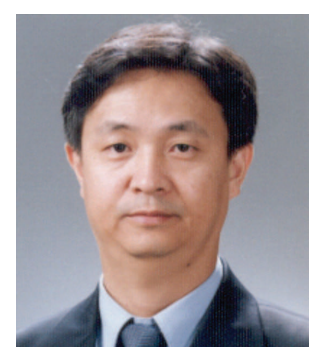

Sung Min Kim received the MS degree in biomedical engineering from University of Iowa in 1991, and the $\mathrm{PhD}$ degree in biomedical engineering from the department of Bioengineering, same university in 1995. He is currently a professor in

Dongguk University and a director of Dongguk Medical Devices Innovation Center. His research interests are in the area of surgery instruments, biomechanics, and bio-health care field. 